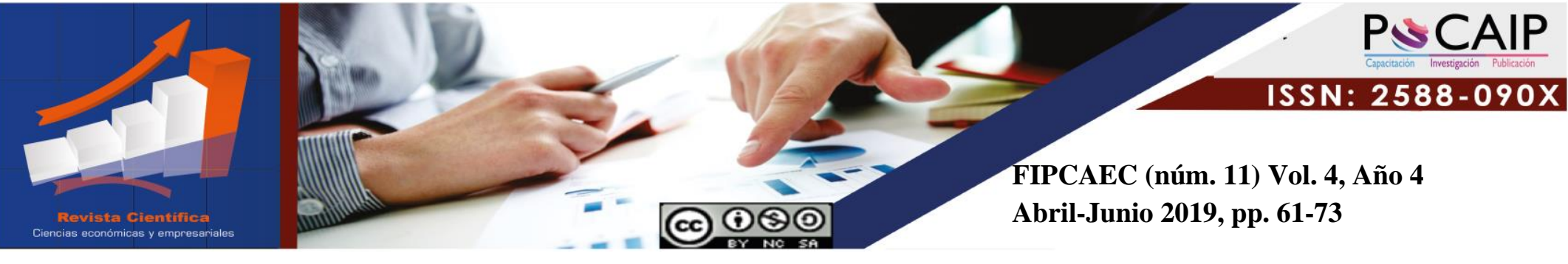

DOI: $10.23857 /$ fipcaec.v4i11.48

\title{
Bauhaus y el desarrollo histórico del diseño gráfico
}

Bauhaus and the historical development of graphic design

\section{Bauhaus e o desenvolvimento histórico do design gráfico}

\author{
Nataly Samara Oyarvide-Estupiñan ${ }^{1}$ \\ nsoyarvide@gmail.com \\ https://orcid.org/0000-0003-2243-8030
}

Correspondencia: nsoyarvide@gmail.com

Recepción: 20/ 01/ 2019 * Aceptación: 18/03/2019 * Publicación: 15 /04/ 2019

1 Ingeniera en Diseño Gráfico Mención Multimedia, Fondos de terceros y seguro de desempleo del Instituto Ecuatoriano de Seguridad Social, Esmeraldas, Ecuador. 


\title{
Resumen
}

La combinación de la academia de Bellas Artes con la Escuela de Artes y Oficios, posterior a la coordinación de Gropius, con la intención de unificar las actividades y eliminar las contradicciones entre artistas y artesanos, permitiendo grandes cambios en el desarrollo histórico del diseño gráfico. Con base en este postulado, este artículo tuvo como objetivo estudiar la escuela de Bauhaus y el desarrollo histórico del diseño gráfico. Para ello se consideró una metodología de tipo hermenéutica y cuyo análisis teórico permitió generar entre sus resultados que existió simultaneidad del desarrollo la escuela de Bauhaus en Alemania, en Francia y otros países europeos optaron por un movimiento distinto. Después de la guerra, el estilo decorativo del modernismo empezó a sustituirse por trazos más simples y rectilíneos, más en armonía con la estética plana y el menor coste de los diseños industriales.

Palabras clave: Bauhaus; desarrollo histórico; diseño gráfico; arte y guerra.

\begin{abstract}
The combination of the Academy of Fine Arts with the School of Arts and Crafts, following the coordination of Gropius, with the intention of unifying activities and eliminating contradictions between artists and craftsmen, allowing great changes in development historical graphic design. Based on this postulate, this article aimed to study the Bauhaus school and the historical development of graphic design. For this purpose, it was considered a hermeneutic methodology and whose theoretical analysis allowed to generate among its results that there was concurrency of the bauhaus school in Germany, in France and other European countries opted for a different movement. After the war, the decorative style of modernism began to be replaced by simpler, more straightened strokes, more in harmony with the flat aesthetics and lower cost of industrial designs.
\end{abstract}

Keywords: Bauhaus; development; graphic design; art and war. 


\section{Resumo}

A combinação da Academia de Belas Artes com a Escola de Artes e Ofícios, após a coordenação de Gropius, com a intenção de unificar atividades e eliminar contradições entre artistas e artesãos, permitindo grandes mudanças no desenvolvimento histórico do design gráfico. Com base neste postulado, este artigo teve como objetivo estudar a escola Bauhaus e o desenvolvimento histórico do design gráfico. Para isso, considerou-se uma metodologia hermenêutica e cuja análise teórica permitiu gerar entre seus resultados a simultaneidade do desenvolvimento da escola Bauhaus na Alemanha, na França e em outros países europeus que optaram por um movimento diferente. Após a guerra, o estilo decorativo do modernismo começou a ser substituído por linhas mais simples e retas, mais em harmonia com a estética plana e com o menor custo dos desenhos industriais.

Palavras-chave: Bauhaus; desenvolvimento histórico; desenho gráfico; Arte e guerra.

\section{Introducción}

La Bauhaus es una escuela de arte, arquitectura y diseño fundada en el año 1919 en Weimar, por el alemán Walter Gropius. Para la Cultura Alemana (2007), ella significa "casa de la arquitectura", término que se gestó al final de la primera Guerra Mundial. Existía temor por la amenaza del comunismo ruso. En tanto existían pensamientos encontrados entre los medios, por una parte se apoyaban las ideas de Marx y Engels generando grandes contradicciones entre los pensamientos de izquierda y derecha. Al respecto, Salvador (2003), expuso este hecho histórico de influencia para la escuela de Bauhaus, dado que llegaban alumnos de todas partes del mundo. Esto, ocasiono que la Alemania conservadora se empezara a sublevar, dado que en sus aulas había extranjeros y mujeres. Sin embargo, un siglo después, el mundo rinde tributo a esta institucion que cambió el rumbo del arte y el diseño.

Se destaca, para Raquejo (2013), que la mayor influencia de esta escuela fue el modernismo, ya desde la década 1880 este movimiento cultural ya había hecho su presencia en Alemania antes de la guerra mundial, a pesar del exagerado conservadurismo que imperaba. Por otra parte, apuntan que, las innovaciones en el diseño se asociaban con Gropius y la Bauhaus, así como las formas radicalmente simplificadas, la racionalidad, funcionalidad y la idea de que la producción en masa 
era conciliable con el espíritu artístico individual, entendiendo que ya se estaban dando en Alemania, mucho antes de que la Escuela la Bauhaus fuera fundada. Estas posiciones teóricas, permitieron estudiar la escuela de Bauhaus y el desarrollo histórico del diseño gráfico.

\section{Desarrollo}

Gómez, C. (2007), explica que la escuela de Bauhaus alcanzo su fama por el enfoque del diseño que publicó y enseñó, la cual funciono como una escuela alemana de arte operacional entre 1919 a 1933 y que combina la artesanía y las Bellas Artes. No obstante, fue fundada con la idea de crear obras de arte total, en la que se incluyeran todas las artes, entre ellas la arquitectura, las cuales serían eventualmente llevadas de manera conjunta. Por lo tanto, el estilo Bauhaus más tarde se convirtió en una de las corrientes más influyentes del diseño moderno, la arquitectura modernista, el arte, el diseño y la enseñanza de la arquitectura, lo que le permitió tener una profunda influencia en posterior en el arte, la arquitectura, el diseño gráfico, el diseño de interiores, el diseño industrial y la tipografía.

\section{Características de la Escuela Bauhaus}

La escuela existió en tres ciudades alemanas, en Weimar de 1919 a 1925, Dessau a partir la 1925 a 1932 y Berlín a partir la 1932 a 1933, bajo tres diferentes directores y arquitectos, Walter Gropius a partir la 1919 a 1928, Hannes Meyer a partir la 1928 a 1930 y Ludwig Mies van der Rohe desde 1930 hasta 1933, cuando la escuela fue cerrada por sus propios líderes bajo presión del régimen Nazi, después de haber sido catalogada como centro del intelectualismo comunista. A pesar de haber sido cerrada, el personal nunca se separó de sus preceptos idealistas, emigrando de Alemania y yéndose a varias partes del mundo. (Campos y col, 2016)

Los cambios de lugar y liderazgo resultaron en un constante cambio de enfoque, técnico, instructores y la política de la Escuela. Por ejemplo, la tienda de cerámica se suspendió cuando la escuela se trasladó de Weimar a Dessau, a pesar de que había sido una fuente de ingresos importante. Igualmente, cuando Mies Van der Rohe asumió el control la escuela en 1930, la transformó en una escuela privada y no le permitiría a ninguno de los partidarios de Hannes Meyer asistir a la misma. (Piquer, 2011) 


\section{Antecedentes de la Escuela Bauhaus}

Expone La escuela fue fundada como una fusión de la gran Escuela Ducal de Artes y oficios y la Academia de Bellas Artes de Weimar. Sus raíces están en la Escuela de Artes y Oficios, fundada por el duque de Saxe en 1906 y dirigida por el arquitecto belga del Art Nouveau Henry Van de Velde. Por otro lado, Van de Velde al ser forzado a dimitir por tener nacionalidad belga, él sugirió a Gropius, Hermann Obrist y a Endell como posibles sucesores. Gropius se hizo el director de la nueva institución de integración de las dos escuelas anteriores llamada la Bauhaus.

Por otra parte, se señala que la derrota de Alemania en la primera Guerra Mundial, la caída de la monarquía alemana y la abolición de la censura permitiendo un aumento de la experimentación radical en todas las artes, que habían sido suprimidas por el antiguo régimen. Una gran mayoría de los alemanes de izquierda fueron influenciados por la experimentación cultural que siguió a la revolución rusa, como el constructivismo. (Fiedler, 2000). Fiedler y col (2000),

\section{Etapas históricas del desarrollo de la escuela de Bauhaus}

Estas etapas, permiten conocer el contacto que marco la escuela de Bauhans en el rumbo del diseño, influencia e importancia en la historia del diseño gráfico.

Primera etapa: esta etapa se dio a inicios de la década de los noventa, en la Alemania se intentó alcanzar el mismo éxito logrado por los ingleses, por lo que al inicio de la década de los noventa se dio impulso a la importación de ideas y reformas desde Inglaterra.

Su estancia tenía como finalidad estudiar las causas del éxito inglés. Gracias a esto se establecieron en el país germano Escuelas de Artes y Oficios en las que diversos artistas modernos que trabajaron como profesores.

En esas escuelas y talleres, para indica Gómez (2007) se producían principalmente enseres de casa, muebles, textiles y utensilios de metal. Estilísticamente, los productos alemanes de final de siglo no tenían parecido alguno con los producidos por los ingleses de las Arts and Crafts, movimiento profundamente arraigado durante el siglo XIX. En esa década de 1890, Alemania 
adelantó a Inglaterra como nación industrializada, asegurándose ese puesto hasta 1914, cuando estalló la Primera Guerra Mundial.

Segunda etapa: durante esta etapa el gobierno alemán coloca la administración de las escuelas de Sächsichen Kunstgewerbeschule y la Sächsischen Hochschule für bildene Kunst a Walter Gropius, quien fundó la Statliches Bauhaus in Weimar, la escuela de arte más moderna en su tiempo. En un manifiesto de la Bauhaus distribuido por toda Alemania, Gropius aclaraba el programa y la meta de la nueva escuela: artistas y artesanos debían trabajar juntos en la construcción del futuro. Gropius declaraba los principios que, en su opinión, deberían regir la escuela desde su inicio. Se refirió a ellos en los siguientes términos retóricos, que evocan un ideal casi cercano a la Edad Media por unificar las artes alrededor del eje de la arquitectura, entre estos, arquitectos, escultores, pintores, todos nosotros debemos regresar al trabajo manual. Establezcamos, por lo tanto, una nueva cofradía de artesanos, libres de esa arrogancia que divide a una clase de la otra y que busca erigir una barrera infranqueable entre los artesanos y los artistas.

A partir de ello, explica se aspiraba construir el nuevo edificio del futuro, que daría cabida a todo a la arquitectura, a la escultura y a la pintura en una sola entidad y que se alzará al cielo desde las manos de un millón de artesanos, símbolo cristalino de una nueva fe que ya llega. Siguiendo las ideas del consejo, construir se convirtió para Gropius en actividad social, intelectual y simbólica. Reconcilió los oficios y especialidades, hasta entonces independientes, uniéndolos como trabajo en común en el que la construcción allana diferencias de condición y acerca a los artistas al pueblo. Todas estas ideas y conceptos se aplicaron en talleres de la misma escuela, donde se daban cita actitudes estéticas en las que alumnos y profesores trabajaban en conjunto.

Ahí se experimentaba con tejidos, trabajo de metales, diseño inmobiliario, fotografía, diseño escenográfico, pintura, escultura y arquitectura. Esto llevó a diseños incipientes, funcionales y eficientes. Figuras del mundo entero se unieron a esta institución pedagógica que orientó el pensamiento de toda una generación de arquitectos, diseñadores y artistas hacia la creación de obras con utilidad social, producidas en serie. 
Tercera etapa: por otro lado, indica Rojas, R (2006), es necesario mencionar el gran impacto pedagógico que esta institución ejerció en los Estados Unidos. Grandes escuelas continuaron con esta manera de enseñar en busca de un nuevo arte útil que ayudara lo más posible a la sociedad en su conjunto. Algunas escuelas con esta tendencia fueron el Instituto Tecnológico de Illinois en Chicago, el Black Mountain College en Carolina del Norte y el Posgrado de la Escuela de Diseño de Harvard, que estuvo bajo la dirección de Gropius de 1938 a 1952.

En el Bauhaus-Archiv Museum für Gestaltung, ubicado en Berlín, actualmente existe un gran número de obras realizadas por los integrantes de esta escuela. El edificio fue diseñado por el mismo Gropius y cuenta con un museo, una colección documental y una biblioteca. Esta organización revela la gran importancia de la escuela, su progreso a lo largo de la historia y la manera en que sirvió como plataforma vital para el desarrollo de nuestro siglo. (Charlotte, 2001)

\section{Principios de la Escuela Bauhaus}

En este sentido, cabe destacar a Charlotte y col (2001) quienes opinan que una de las más importantes clases en la Bauhaus fue la tipografía. De hecho, varios profesores pronto se dieron cuenta del papel esencial de los tipos de comunicación visual efectiva. La Bauhaus se concentró en fuentes simplificadas y evita las representaciones mucho más pesadas de la tipografía estándar alemana de la época.

Así, los diseñadores comenzaron a envolver texto alrededor de objetos y también aprendieron a organizarlo de manera horizontal, vertical y diagonalmente incluso, lo cual no era común en aquel tiempo. También se negaron a combinar los tipos de cajas superiores e inferiores en una misma obra y prefieren el uso de tipografías tipo "sin serifas" (sans-serif). Las innovaciones introducidas por la Bauhaus todavía son muy eficaces en la actualidad. (Charlotte, 2001).

Por lo tanto, un ejemplo de esta tendencia, es el cartel de Barack Obama, durante su campaña electoral en el 2008, quien visitó Berlín y su discurso en la ciudad fue anunciado con este cartel muy similar a los de la escuela alemana. De hecho se puede ver que se caracteriza por las 
palabras en diagonal y una letra mayúsculas sin serifas, igualmente, en el diseño no hay decoraciones inútiles y predominan las líneas simples.

La Bauhaus también demostró un profundo interés por la geometría simple, otra cualidad que hace que sea de gran ajuste para diseño actual en la red. Los estudiantes eran muy bien formados, tomando las ideas de las pinturas de artistas contemporáneos, en este caso de cubistas como Picasso y Gris, adoptando entonces formas similares de ver y percibir la realidad. Para los diseños con tendencia geométrica, comenzaron rompiendo objetos con estas formas, considerando esta técnica como la mejor manera de crear artículos nuevos y más modernos. (Torres, 2007).

En este sentido, explica Toajas (2012) que las formas limpias, abstractas y geométricas fueron utilizadas constantemente para producir nuevas formas más comunes que podrían resaltar la diferencia de las viejas tendencias del Nouveau Art. Un ejemplo de su diseño, fue que en 1925, Marcel Breuer, un miembro de la escuela Bauhaus, diseñó un nuevo modelo de silla, que más tarde fue llamado la "Silla Wassily". Esta silla está formada por unos tubos de metal y bandas de cuero que dan una idea de la fluidez y flexibilidad, siendo el diseñador capaz de realizar un diseño mínimo y fluido que duró años y que todavía es preferido y solicitado hoy en día.

El diseño identidad (ID), explica Estrada (2010), en la web moderna sigue siendo por excelencia del estilo Bauhaus. Ya que este diseño actual es un sitio con lo mínimo y sencillo para resaltar la experiencia del usuario en el proyecto. La cabecera muestra sólo dos letras, "ID”, luego hay una barra de menú transparente que lleva dentro al contenido. Además, siguiendo las tendencias de la Bauhaus, el diseño "Identidad" no utiliza serifas, ni tipografías demasiado complejas.

\section{Metodología}

El artículo destaco una metodología de tipo hermenéutica bajo un enfoque analítica- documental, fundamentada en lo propuesto por Hurtado (2015), quien explica que este tipo de investigación busca analizar un evento y comprenderlo en términos de sus aspectos menos evidentes. Según Hernández y col (2014), la investigación documental es una técnica que consiste en la selección y compilación de información a través de la lectura y crítica de documentos y materiales 
bibliográficos, bibliotecas, bibliotecas de periódicos, centros de documentación e información, que en este caso particular refirió a la Escuela de Bauhaus y el desarrollo histórico del diseño gráfico, es de indicar que este tipo de investigación incluye tanto el análisis como la síntesis.

\section{Reflexiones finales}

La existencia de movimientos distintos, de forma simultaneas el desarrollo de la escuela de Bauhaus y en los países europeos se optó por un movimiento distinto. Asimismo, se develo después de la guerra un estilo decorativo del modernismo, iniciado con la sustitución de trazos más simples y rectilíneos, con mayor armonía en la estética plana y en menor coste de los diseños industriales.

Por otro lado, en el modernismo se reflejan las líneas duras y la solidez de las formas y la aparición de elementos monumentales en sus composiciones, así como las del estilo racionalista en la aparición de elementos monumentales en sus composiciones. Por otro lado, se puede observar en los diseños de las innovaciones tecnológicas de su época, tales como la aviación moderna, la iluminación eléctrica, la radio, el revestimiento marino y los rascacielos, en la que dominaban las formas geométricas y de la simetría.

Cabe destacar, que es en el siglo XVI, cuando se empiezan a presentar las primeras consecuencias graficas de las iniciativas tomadas en esta época, en la qiue aparecieron innovadores diseños de tipos, normas de composición, ilustraciones y encuadernaciones, conjuntamente con representantes de gran prestigio.

Por otro lado, cabe destacar la influencia que tuvieron hechos como la Revolución Francesa y la Revolución Industrial, para el desarrollo del comercio y de la actividad publicitaria. Además, es importante tomar en cuenta que muchos aspectos políticos, sociales y religiosos de cada época, han influido en la evolución o retroceso en lo que concierne a los trabajos impresos, desde la creación de los tipos de letras, el trabajo tipográfico, hasta la aparición de los libros, periódicos, enciclopedias y carteles. 
Se pudo deducir, que a comienzo del siglo $\mathrm{XX}$ se vio la urgencia de generar masivamente productos que satisficieran las necesidades de un mercado cada vez más amplio y exigente. Es decir, que requirieran productos que pudieran ser adquiridos por cualquier persona, evitando así que circularan solamente en una clase social o en una elite reducida. La demanda de más y mejores bienes impulsó y obligó a los talleres de arte y de mano de obra a convertirse en diseñadores industriales.

Por otra parte, debido a los problemas sociales y económicos del momento, se trataban de crear productos que fueran funcionales pero que a la vez presentaran un diseño atractivo para el mercado, manteniendo bajos los costos de producción. Para lograrlo, se empezó a producir con materiales comunes y baratos como el metal, el vidrio, el cristal y la madera, entre otros. Se creía que las formas y los colores básicos representaban un precio industrialmente más económico, por lo que las formas del círculo, el cuadrado y el triángulo fueron tomadas como puntos de partida.

En las clases relacionadas con la forma se empezaba a trabajar con dichas figuras elementales y a cada una de ellas se le atribuía un carácter determinado. Así, el círculo era "fluido y central", el cuadrado resultaba "sereno" y el triángulo, "diagonal". Más adelante, en el arte y en la escultura de Bauhaus se tomó este mismo concepto con el que trabajaron Wassily Kandinsky, Paul Klee y Johannes Itten. A este nuevo desarrollo, representado principalmente en Holanda y Alemania, se le llamó funcionalidad moderna o modernidad internacional.

De allí, que posterior a la fundación de la escuela de Bauhaus, que se pudo identificar la innovadora producción estética con la maquinaria industrial. Sus tendencias fundamentales se reducen a la ruptura con lo tradicional y con los estilos preestablecidos; el predominio de la función sobre la forma; la interrelación estrecha entre, por un lado, la arquitectura y el diseño y, por otro, las ciencias aplicadas; la adecuación de la vivienda a los recursos y necesidades humanas y una efectiva planificación urbana.

Es de destacar, que la filosofía de Gropius se basaba en integrar todas las artes con la tecnología moderna y unirlas con el fin de obtener un diseño disponible para todos los niveles socioeconómicos. Los productos resultantes se alejaban mucho de la clásica ornamentación excesiva. Por el contrario, poseían líneas limpias y claras, formas geométricas sencillas y, de 
manera característica, daban la impresión de estar hechas industrialmente y no a mano. Fue entonces cuando se empezó a hablar de hacer diseños industriales en forma positiva. La teoría de la Bauhaus sobre la enseñanza del diseño incluía un artista y un técnico en cada estudio de clase. De esta manera, los alumnos usaban su creatividad de forma libre y simultáneamente, aprendían la técnica. Los alumnos eran requeridos en los talleres por periodos cortos, con el fin de que entendieran la tecnología para la que posteriormente diseñarían.

\section{Referencias}

1. Cabrejas, M. (2009). Fotografía de ficción en Japón en el siglo XIX. Recreaciones de escenas para el mercado occidental. Anales de Historia del Arte, 19. pp. 257-270. ISSN 0214-6452

2. Campos, A., Gaitán, C.,García, F., De Haro, N., Mateo, L., Moreno, F., Murga,I., Pérez, A.,Sainz, Ó y Cabañas, M. (2016). Escenarios de guerra: paseando por Madrid a través de su memoria. Otros. Doce Calles, Madrid.

3. Carmel, J y Philippe, J. (1999). Starck. Editorial Calton. Londres.

4. Costa, J. (2012). El DirCom, hoy: dirección y gestión de la comunicación en la nueva economía. Barcelona: Costa Punto Com.

5. Cultura

Alemana

(2007).

Recuperado

en: http://www.germanculture.com.ua/spanish/esindex.html

6. Charlotte y Peter Fiell. (2001). Diseño del siglo XX. Editorial Taschen. Colonia.

7. Estrada, S. (2010) Desarrollo de un proyecto gráfico. Barcelona: Index Book.

8. Fiedler, J y Feierabend, P. (2000). Bauhaus. Editorial Konemann. Colonia.

9. Gómez, C. (2007). Bauhaus. Recuperado en: http://www.chgblog.com/bauhaus.

10. Hernández R, Fernández C y Baptista, P (2014). Metodología de la Investigación. Chile: McGraw - Hill. 
11. Rojas, $\mathrm{R}$ (2006). La Bauhaus. Recuperado en: http://www./la-bauhaus/labauhaus.shtml?monosearch.

12. Salvador, J (2003). Trayectoria del escultor Eloy Palacios hasta 1883. Argos (Caracas) (38). pp. 113-147. ISSN 0254-1637

13. Toajas, M., Paniagua, J.,Silva, N., Fuentes, S y Guerrero, Eva (2012) Glosario visual de técnicas artísticas de la antigüedad a la Edad Moderna. Arquitectura. Pintura. Artes gráficas. Artes suntuarias. Escultura. Manual. Universidad Complutense - Vicerrectorado de Desarrollo y Calidad de la Docencia, Madrid.

14. Torres, E. (2007). El diseño gráfico delineado en el tiempo. Clase magistral para el décimo semestre de Publicidad de la Universidad Alejandro de Humboldt. CaracasVenezuela.

\section{References}

1. Cabrejas, M. (2009). Fiction photography in Japan in the 19th century. Recreations of scenes for the western market. Annals of Art History, 19. pp. 257-270. ISSN 0214-6452

2. Campos, A., Gaitán, C., García, F., De Haro, N., Mateo, L., Moreno, F., Murga, I., Pérez, A., Sainz, Ó and Cabañas, M . (2016). War scenarios: walking through Madrid through his memory. Others. Twelve Streets, Madrid.

3. Carmel, J and Philippe, J. (1999). Starck Calton Publishing. London.

4. Costa, J. (2012). The DirCom, today: communication management and management in the new economy. Barcelona: Costa Punto Com.

5. German Culture (2007). Retrieved from: http://www.germanculture.com.ua/spanish/esindex.html

6. Charlotte and Peter Fiell. (2001). 20th century design Taschen publishing house. Suburb.

7. Estrada, S. (2010) Development of a graphic project. Barcelona: Index Book.

8. Fiedler, J and Feierabend, P. (2000). Bauhaus Konemann editorial. Suburb. 


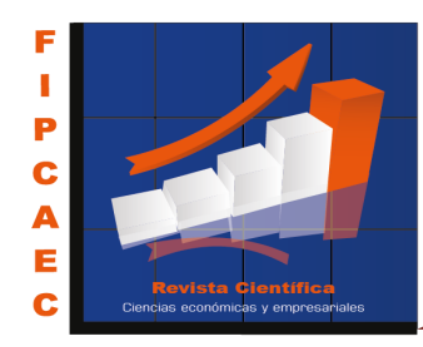

9. Gómez, C. (2007). Bauhaus Recovered at: http://www.chgblog.com/bauhaus.

10. Hernández R, Fernández C and Baptista, P (2014). Investigation methodology. Chile: McGraw - Hill.

11. Rojas, $\mathrm{R}$ (2006). The Bauhaus Retrieved from: http://www./la-bauhaus/labauhaus.shtml?monosearch.

12. Salvador, J (2003). Trajectory of the sculptor Eloy Palacios until 1883. Argos (Caracas) (38). pp. 113-147. ISSN 0254-1637

13. Toajas, M., Paniagua, J., Silva, N., Fuentes, S and Guerrero, Eva (2012) Visual glossary of artistic techniques from antiquity to the Modern Age. Architecture. Painting. Graphic arts. Sumptuary arts. Sculpture. Manual. Universidad Complutense - Vice-Rectorate for Development and Quality of Teaching, Madrid.

14. Torres, E. (2007). The graphic design outlined in time. Master class for the tenth semester of Advertising at the Alejandro de Humboldt University. Caracas Venezuela. 